\title{
DIFFERENT SCATTERING REGIMES IN TWO-DIMENSIONAL BOSE-EINSTEIN CONDENSATES
}

\author{
B. Tanatar \\ Department of Physics, \\ Bilkent University, \\ 06533 Ankara, Turkey
}

\begin{abstract}
Motivated by the recent efforts to produce low-dimensional condensates, we study the ground-state density profiles of two-dimensional Bose-Einstein condensed atoms at zero temperature within a mean-field theory. The interplay between the tight harmonic confinement in the axial direction and collisional properties of the condensate atoms help identify three distinct regimes of experimental interest. Each regime is described by a different atom-atom coupling which depends on the density of the condensate as the system starts to be influenced by two-dimensional collisions. We trace the regions of experimentally accessible system parameters for which the crossover between different dimensionality behaviors in the scattering properties may become observable.
\end{abstract}

Keywords: Bose-Einstein condensates, low-dimensional systems

\section{Introduction}

The observation of Bose-Einstein condensation Bose-Einstein condensation (BEC) in externally confined atomic vapors $[1,2,3]$ has stimulated a big interest in the theoretical and experimental work on interacting systems of bosons. boson The thermodynamic, ground-state static and dynamic properties of condensates are extensively investigated and the main results are compiled in a number of reviews. $[4,5,6,7]$ Most of the excitement stems from the possibility of understanding properties of a macroscopic quantum state. Other than the fundamental physics considerations, the Bose-Einstein condensed systems offer interesting applications of atom laser laser and atom optics.

The BEC phenomenon in low dimensional and particularly in two-dimensional (2D) systems has attracted considerable amount of interest from the point of view of understanding effects of dimensionality. As the homogeneous 2D system of bosons boson would not undergo BEC at a finite temperature, the prospects of observing BEC in systems with an external potential $[8,9]$ provide a strong motivation for such investigations. It was argued by Mullin[10] that 
BEC is not possible for strictly 2D systems even in a trapping trapping potential in the thermodynamic limit. However, by varying the trapping field so that it is very narrow in one direction, it should be possible to separate the singleparticle states of the oscillator potential into well-defined bands, and occupying the lowest band should produce an effectively two-dimensional system. Growing number of experiments[11,12, 13, 14, 15, 16] exploring the possibility of realizing quasi-one-dimensional (Q1D) and quasi-two-dimensional (Q2D) trapped atomic gases culminated in the recent experiments of Görlitz et al.[17] in achieving low-dimensional condensates. and measurements on the BEC transition temperature and other properties are expected to follow.

The studies on the BEC in 2D systems can be broadly divided into two categories. In the first group the interaction effects are treated parametrically without reference to the actual interaction potential or the scattering length which describes it as in the 3D formulation of the interacting boson boson condensates.[18, 19, 20, 21] Included in the same spirit of calculations there has been path integral Monte Carlo simulations[22] at finite temperature to give indications of a BEC transition in 2D systems. In the second group, some effort is made to relate the $2 \mathrm{D}$ interaction strength to the $3 \mathrm{D}$ scattering length $[23,24,25]$ or to solve the scattering problem in strictly $2 \mathrm{D}$ to obtain the relevant dependence of the interaction coupling on the scattering length. Kim et al.[26] using the scattering theory in $2 \mathrm{D}$, found that the interaction strength depends logarithmically on the scattering length. Shevchenko[27, 28] in a series of papers studied interacting 2D Bose gas in a nonuniform field arriving at the conclusion even though a Bose-Einstein condensation Bose-Einstein condensation does not take place, the system exhibits superfluidity. Recently, Kolomeisky et al.[29] in their treatment of low-dimensional Bose liquids suggested a modified form for the mean-field description of 2D condensates. Lieb, Seiringer, and Yngvason [30] have rigorously analyzed this and related approximations as applied to the practical cases of interest. Petrov and coworkers[31,32] using scattering theory arguments obtained a slightly more detailed form of the effective interaction coupling for Q2D systems that distinguishes different density regimes. Similar results are also obtained by Lee et al.[33] within a many-body $T$-matrix approach.

In this paper we introduce various interaction coupling models to describe the pancake shaped 2D condensates formed in highly anisotropic traps. Our central aim is to calculate the equilibrium density (or wavefunction) profiles of the condensate with different choices of system parameters such as anisotropy in the trap frequencies and scattering length. Our calculations show that with increasing anistropy the condensate first becomes $2 \mathrm{D}$ with regard to the confinement and then also in its collisional properties. We find that these different regimes may be identified by measuring the size of the cloud in the radial plane and we characterize the crossover regime in terms of the relevant physical parameters. 


\section{Theory}

The ground-state properties of a condensed system of bosons boson at zero temperature are described by the Gross-Pitaevskii equation which is a nonlinear Schrödinger equation. In the presence of external trap potentials, therefore an inhomogeneous Bose system, it is useful to adopt the local-density approximation which regards the system locally homogeneous. We consider a dilute Bose-condensed gas in anisotropic harmonic confinement characterized by the frequencies $\omega_{\perp}$ and $\omega_{z}=\lambda \omega_{\perp}$ with $\lambda>>1$ to yield a pancake shaped condensate. The mean-field energy functional in the local-density approximation can very generally be written as $[34,35,36]$

$$
E=\int d \mathbf{r}\left[\frac{\hbar^{2}}{2 m}|\nabla \psi|^{2}+V_{\mathrm{ext}}(\mathbf{r})|\psi|^{2}+\epsilon(\rho)|\psi|^{2}\right],
$$

where $\epsilon(\rho)=g \rho / 2$ is the ground-state energy (per particle) of the homogeneous system, and $\rho=|\psi|^{2}$ is the density. The coupling parameter $g$ can depend on the condensate density, $g=g(\psi)$. Variation of the energy functional with respect to $\psi^{*}$, subject to the normalization condition $\int d \mathbf{r}|\psi|^{2}=N$, yields the nonlinear Schrödinger equation

$$
-\frac{\hbar^{2}}{2 m} \nabla^{2} \psi+V_{\mathrm{ext}}(\mathbf{r}) \psi+\frac{\partial[\epsilon(\rho) \rho]}{\partial \rho} \psi=\mu \psi,
$$

where $\mu$ is the chemical potential. The local-density approximation was used by Fabrocini and Polls[35] and Nunes[34] to study the high density effects in 3D condensates, by making use of the 3D homogeneous hard-sphere Bose gas results from perturbation theory. These studies showed that the modifications to GP equation become important as the number of particles $N$ and the hard-sphere radius $a$ are increased making the system less dilute.

To explore different regimes of $2 \mathrm{D}$ condensates we consider various models. [37] In the first approach, we consider a condensate whose third dimension is of the order of the harmonic confinement length $a_{z}=\left(\hbar / m \omega_{z}\right)^{1 / 2}$. If the condition $a<<a_{z}$ holds, the system undergoes collisions in three dimensions and the coupling constant we use in the 2D GP equation is $g_{Q 3 D}=g_{3} D \mid \phi(z=$ $0)\left.\right|^{2}$ where $\phi(z=0)=\left(2 \pi a_{z}^{2}\right)^{-1 / 4}$ is the axial ground state ground state wavefunction evaluated at $z=0$. It takes the form

$$
g_{Q 3 D}=2 \sqrt{2 \pi} \frac{\hbar^{2}}{m} \frac{a}{a_{z}}
$$

reflecting the geometrical effects of the reduced dimensionality. A number of authors $[23,24,25]$ have used this coupling constant to describe 2D condensates.

When the anisotropy further increases and the scattering length $a$ becomes of the order of $a_{z}$, the collisions among the atoms start to be influenced by 
the presence of the trap in the tight $z$-direction. Petrov et al.[31, 32] and Lee et al.[33] studied the scattering problem in quasi-two-dimension (Q2D) of a system harmonically confined in the $z$-direction and homogeneous in the perpendicular direction, to obtain the following expression for the coupling strength

$$
g_{Q 2 D}=\frac{2 \sqrt{2 \pi}\left(\hbar^{2} / m\right)\left(a / a_{z}\right)}{1+\frac{\left(a / a_{z}\right)}{\sqrt{2 \pi}}\left|\ln \left(g_{Q 2 D} \rho\left(2 \pi m / \hbar^{2}\right) a_{z}^{2}\right)\right|} .
$$

In the Q2D regime the coupling strength depends on the condensate density $\rho$. Furthermore, $g_{Q 2 D}$ is given by an implicit relation which has to be determined numerically during the solution of the 2D GP equation. If we make a zero-order approximation for $g_{Q 2 D}$ appearing in the logarithm, i.e. $g_{Q 2 D} \approx 2 \sqrt{2 \pi}\left(\hbar^{2} / m\right)\left(a / a_{z}\right)$, then the expresion for Q2D coupling strength simplifies to

$$
g_{Q 2 D}=\frac{2 \sqrt{2 \pi}\left(\hbar^{2} / m\right)\left(a / a_{z}\right)}{1+\frac{\left(a / a_{z}\right)}{\sqrt{2 \pi}}\left|\ln \left(2(2 \pi)^{3 / 2} \rho a a_{z}\right)\right|} .
$$

In our numerical calculations we have found that the above implicit and explicit forms of $g_{Q 2 D}$ slightly change the wavefunction profiles only for very large values of asymmetry parameter $\lambda$.

The strictly $2 \mathrm{D}$ regime is attained when the collisional properties are such that $a>>a_{z}$. harmonic isotropic trapping potential $V_{\mathrm{ext}}(\mathbf{r})=m \omega_{\perp}^{2} r^{2} / 2$. Perturbation theory calculations for a homogeneous system of $2 \mathrm{D}$ hard-disk bosons boson yield[38]

$$
\epsilon(\rho)=\frac{\hbar^{2}}{2 m} \frac{4 \pi \rho}{\left|\ln \rho a^{2}\right|},
$$

for the ground-state energy (per particle). The corresponding mean-field equation for the condensate wave function thus reads

$$
-\frac{\hbar^{2}}{2 m} \nabla^{2} \psi(r)+\frac{1}{2} m \omega_{\perp}^{2} r^{2} \psi(r)+\frac{\hbar^{2}}{2 m} \frac{8 \pi}{\left|\ln \psi^{2} a^{2}\right|}|\psi(r)|^{2} \psi(r)=\mu \psi,
$$

from which the $2 \mathrm{D}$ intertaction coupling can be identified

$$
g_{2 D}=\frac{4 \pi \hbar^{2}}{m} \frac{1}{\left|\ln \left(\rho a^{2}\right)\right|}
$$

The energy functional corresponding to this equation has also been suggested by Shevchenko[28] and more recently by Kolomeisky et al.[29]

We note that the ensuing GP equation in 2D is quite different than its 3D counterpart, in that the dimensionless interaction term $g_{Q 2 D}$ or $g_{2 D}$ not only 
depends on the hard-disk radius $a$ logarithmically, but it also depends on $|\psi|^{2}$, making the GP equation highly nonlinear. In 3D the density dependent interactions arise as corrections or modifications to the GP equation, whereas in 2D the mean-field interaction is density dependent.

In the previous applications $[34,35,39]$ of the local-density approximation in $3 \mathrm{D}$ condensates, effects beyond the mean-field theory (GP equation) has been explored by including higher order terms in the homogeneous energy density $\epsilon(\rho)$. In 2D, the correct mean-field description is given by Eq. (2). To go beyond the mean-field theory one would have to use the higher order terms in the perturbation series for $\epsilon(\rho)$. Another possible correction to the meanfield description has been suggested by Andersen and Haugerud.[40] In their treatment the kinetic energy functional is modified to include a gradient term

$$
E_{\text {kin }}=\frac{\hbar^{2}}{2 m} \int d \mathbf{r}\left[1-\frac{2}{3} \frac{1}{\left.|\ln | \psi\right|^{2} a^{2} \mid}\right]|\nabla \psi|^{2} .
$$

In our numerical calculations to be presented and discussed in the next section, we have tested the significance of corrections brought by the gradient term. We have found that it has negligible effect on the condensate profile $\psi(r)$ and other physical quantities for the range of $N$ and scattering length values we examined.

\section{Results and Discussion}

We solve the Gross-Pitaevskii equation given in Eq. (2) for three models of $g(\psi)$ numerically using the steepest descent method $[35,41]$ which is known to produce accurate results. A further check of our numerical calculations is provided by the virial relation. Under the scaling transformation $\mathbf{r} \rightarrow \lambda \mathbf{r}$, so that $\psi(\mathbf{r}) \rightarrow \psi(\mathbf{r}) / \lambda$, and using the variational nature of the energy we obtain

$$
E_{\text {kin }}-E_{\text {ext }}+E_{\text {int }}-\frac{1}{2} \int d \mathbf{r} \frac{d g(\psi)}{d \psi}|\psi(\mathbf{r})|^{5}=0 .
$$

In this form of the virial relation, we have omitted the gradient correction to the kinetic energy, since numerically it is found to be negligibly small. Our solution of the GP equation satisfies the virial relation to a high degree.

In the following we illustrate the ground-state density $|\psi(r)|^{2}$ predicted by the three models. We first consider the parameters as appropriate for ${ }^{23} \mathrm{Na}$ atoms in the experiment of Görlitz et al.[17] taking $N=5 \times 10^{5}, \lambda=26.33$, and $a=2.8 \mathrm{~nm}$. For these parameters the condensate appears as $2 \mathrm{D}$ due to its confinement characteristics $\left(\mu \simeq 2.08 \hbar \omega_{z}\right.$ ) but the collisions have a 3D nature $\left(a / a_{z} \simeq 3.8 \times 10^{-3}\right)$. Density profiles in this Q3D regime are displayed in Fig. 1 for all three models. It is observed that Q3D and Q2D interaction models yield almost identical predictions (solid and dotted lines). The fully 2D model, 


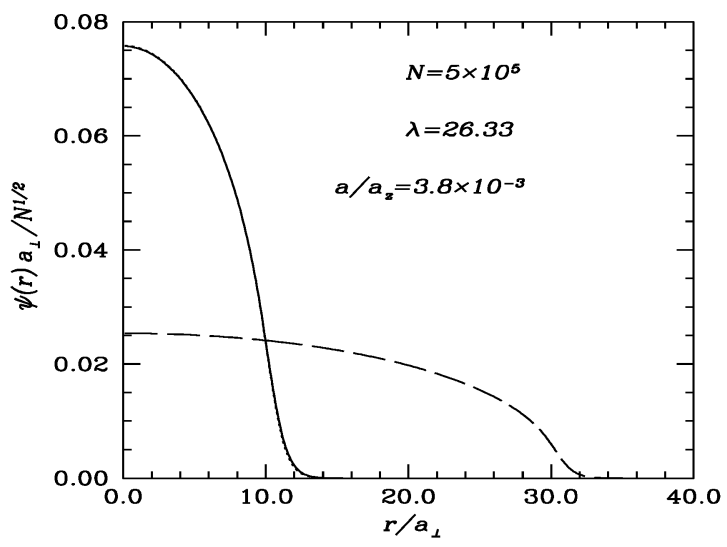

Figure 1. The condensate density $|\psi(r)|^{2}$ (in units of $N / a_{\perp}^{2}$ ) as a function of the radial distance for a system of $N=10^{5}$ atoms, $a / a_{z}=3.8 \times 10^{-3}$ and $\lambda=26.33$ from the numerical solution of GP equation for the three models: Q3D (dotted line), Q2D (solid line), and 2D (dashed line).

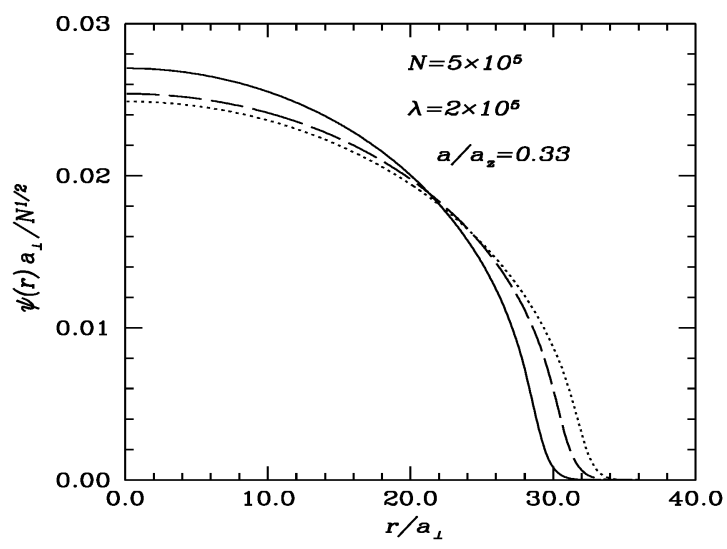

Figure 2. The same as in Fig. 1, for $a / a_{z}=0.33$ and $\lambda=2 \times 10^{5}$. 


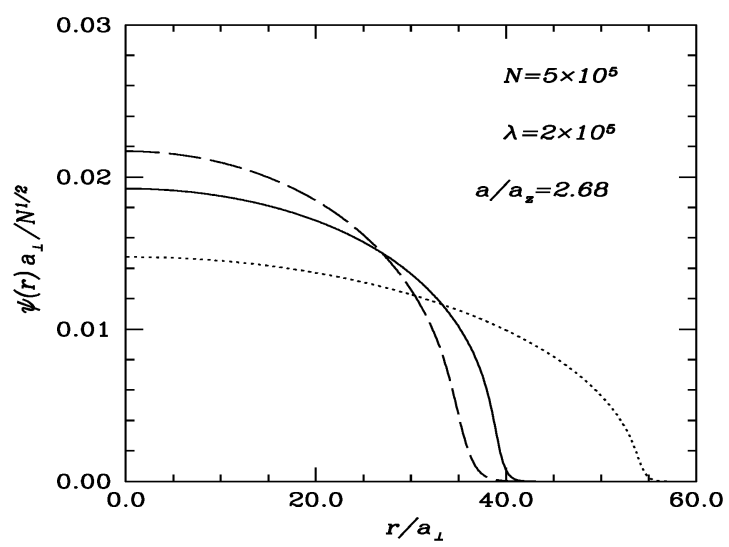

Figure 3. The same as in Fig. 1, for $a / a_{z}=2.68$ and $\lambda=2 \times 10^{5}$.

on the other hand, produces a quantitatively very different density profile with a much larger chemical potential $\left(\mu \simeq 17.8 \hbar \omega_{z}\right)$. It is evident that $2 \mathrm{D}$ model is not applicaple to the current experimental situation.[17]

In the second case we increase the anisotropy parameter to a much larger value $\lambda=2 \times 10^{5}$ and make the choice $a / a_{z}=0.33$ for the scattering length. These parameters correspond to the regime of crossover in the scattering properties from $3 \mathrm{D}$ to $2 \mathrm{D}$ in a condensate where the motion in the third dimension is completely frozen by the confinement effects $\left(\mu \simeq 0.002 \hbar \omega_{z}\right)$. As shown in Fig. 2 in the crossover regime the three models predict comparable shapes of the condensate cloud indicating the approach to $2 \mathrm{D}$ collisions.

Finally, we further increase the value of the scattering length to $a / a_{z}=2.68$, keeping the same values of $\lambda$ and $N$ as the previous case. For this choice of parameters the collisions are truly 2D and the strictky 2D model should be relevant in predicting an accurate profile of the condensate cloud. The other models would overestimate the width of $\psi(r)$. Our results confirming this scenario are shown in Fig. 3. 


\section{Summary and Concluding Remarks}

In summary we have considered Bose-Einstein condensates Bose-Einstein condensation confined in pancake shaped traps at zero temperature within a mean-field description. Three different physical regimes are identified for the scattering properties of the condensate in terms of the anisotropy of the trap; (i) a Q3D regime where the axial dimension of the condensate is much larger than the scattering length and the collisions are as in a 3D condensate,

(ii) a Q2D regime where the tight harmonic confinement in the $z$-direction begins to influence the scattering events,

(iii) a strictly $2 \mathrm{D}$ regime where collisions are restricted to the $x-y$ plane.

The interaction coupling is different in the three cases and a logarithmic dependence on the density arises as the $2 \mathrm{D}$ effects start to affect the scattering properties. We have adopted a local-density approximation to introduce the appropriate coupling strength into the energy functional of the condensate and to obtain a GP equation for its in-plane wavefunction.

Our results should be relevant to the experimental efforts aiming to produce low-dimensional condensates. The results of our calculations show that the different collisional regimes are reflected in the width of the cloud. Observable properties are expected for flat condensates not only due to the geometric confinement effects (i.e. when $\mu<\hbar \omega_{z}$ ) but also when the condition $a>a_{z}$ holds. The strictly $2 \mathrm{D}$ limit can be reached by making the tight confinement very large and by increasing the scattering length. Recent experiments of Cornish et al. [42] have demonstrated the feasibility of tuning the scattering length through Feshbach resonances. This opens the possibility of studying the effects of interactions and regimes those described beyond the Gross-Pitaevskii equation more systematically. To assess the importance of interactions and high density effects Monte Carlo simulations would be useful as a test of range of validity of the mean-field and local-density approximations. Our calculations should be the starting point for finite temperature studies in which the role of interactions with the non-condensed thermal particles and phase correlations at increased temperatures may be addressed.

\section{Acknowledgments}

This work was partially supported by the Scientific and Technical Research Council of Turkey (TUBITAK), by NATO-SfP, by the Turkish Department of Defense, and by Turkish Academy of Sciences (TUBA). Fruitful discussions with Professor M. P. Tosi, and Drs. A. Minguzzi and P. Vignolo are kindly acknowledged. 


\section{References}

[1] Anderson M. H., Ensher J. R., Matthews M. R., Wieman C. E., and Cornell E. A., Science 269, 198 (1995).

[2] Davis K. B., Mewes M.-O., Andrews M. R., van Druten N. J., Durfee D. S., Kurn D. M., and Ketterle W. (1995) Bose-Einstein condensation of Sodium atoms, Phys. Rev. Lett. 75, 3969.

[3] Bradley C. C., Sackett C. A., and Hulet R. G. (1997) Bose-Einstein condensation of Lithium: observation of limited condensate number, Phys. Rev. Lett. 78, 985.

[4] Dalfovo F., Giorgini S., Pitaevskii L. P., and Stringari S. (1999) Theory of Bose-Einstein condensation in trapped gases, Rev. Mod. Phys. 71, 463.

[5] Parkins A. S. and Walls D. F. (1998) The physics of trapped dilute-gas Bose-Einstein condensates, Phys. Rep. 303, 1.

[6] Leggett A. J. (2001) Bose-Einstein condensation in the alkali gases: some fundamental concepts, Rev. Mod. Phys. 73, 307.

[7] Courtieille Ph. W., Bagnato V. S., and Yukalov V. I. (2001) Bose-Einstein condensation of trapped atomic gases, Laser Phys. 11, 659.

[8] Bagnato V. and Kleppner D. (1991) Bose-Einstein condensation in low-dimensional traps, Phys. Rev. A 44, 7439.

[9] De Groot S. R., Hooyman G. J., and ten Seldam C. A. (1950) On the Bose-Einstein condensation, Proc. R. Soc. London Ser. A 203, 266.

[10] Mullin W. J. (1998) A study of Bose-Einstein condensation in a two-dimensional trapped gas, J. Low Temp. Phys. 110, 167.

[11] Hinds E. A., Boshier M. G., and Hughes I. G. (1998) Magnetic waveguide for trapping cold atom gases in two-dimensions, Phys. Rev. Lett. 80, 645.

[12] Gauck H., Hartl M., Schneble D., Schnitzler H., Pfau T., and Mlynek J. (1998) Quasi-2D gas of laser cooled atoms in a planar matter waveguide, Phys. Rev. Lett. 81, 5298.

[13] Vuletić V., Kerman A. J., Chin C., and Chu S. (1999) Observation of low-field Feshbach resonances in collisions of Cesium atoms, Phys. Rev. Lett. 82, 1406.

[14] Bouchoule I., Perrin H., Kuhn A., Morinaga M., and Salomon C. (1999) Neutral atoms prepared in Fock states of a one-dimensional harmonic potential, Phys. Rev. A 59, 8.

[15] Dettmer S., Hellweg D., Ryytty P., Arlt J. J., Ertmer W., Sengstock K., Petrov D. S., Shlyapnikov G. Y., Kreutzmann H., Santos L., and Lewenstein M. (2001) Observation of phase fluctuations in elongated Bose-Einstein condensates, Phys. Rev. Lett. 87, 160406.

[16] Burger S., Cataliotti F. S., Fort C., Maddaloni P., Minardi F., and Inguscio M. (2002) Quasi-2D Bose-Einstein condensation in an optical lattice, Europhys. Lett. 57, 1.

[17] A. Görlitz, J. M. Vogels, A. E. leanhardt, C. Raman, T. L. Gustavson, J. R. Abo-Shaeer, A. P. Chikkatur, S. Gupta, S. Inouye, T. P. Rosenband, D. E. Pritchard, and W. Ketterle, (2001) Realization of Bose-Einstein condensates in lower dimensions, Phys. Rev. Lett. 87, 130402.

[18] Haugset T. and Haugerud H. (1998) Exact diagonalization of the Hamiltonian for trapped interacting bosons in lower dimensions, Phys. Rev. A 57, 3809.

[19] Bayindir M. and Tanatar B. (1998) Bose-Einstein condensation in a two-dimensional, trapped, interacting gas Phys. Rev. A 58, 3134.

[20] Kim J. G. and Lee E. K. (1999) Thermodynamic properties of trapped, two-dimensional interacting Bose gases in Hartree-Fock approximation, J. Phys. B 32, 5575. 
[21] Adhikari S. K. (2000), Phys. Lett. A 265, 91.

[22] Heinrichs S. and Mullin W. J. (1998), J. Low Temp. Phys. 113, 231.

[23] Bhaduri R. K., Reimann S. M., Viefers S., Choudhury A. G., and Srivastava M. K. (2000) The effect of interactions on Bose-Einstein condensation in a quasi two-dimensional harmonic trap, J. Phys. B 33, 3895.

[24] Salasnich S., Parola A., and Reatto L. (2002) Effective wave equation for the dynamics of cigar-shaped and disk-shaped Bose condensates, Phys. Rev. A 65, 043614.

[25] Kim J. G., Kang K. S., Kim B. S., and Lee E. K. (2000) Hartree-Fock calculation for trapped, two-dimensional interacting Bose gases of finite size, J. Phys. B 33, 2559.

[26] Kim S.-H., Oh S. D., and Jhe W. (2000) Two-dimensional condensation of dilute Bose atoms in a harmonic trap, J. Kor. Phys. Soc. 37, 665.

[27] Shevchenko S. I. (1991) Theory of two-dimensional superfluidity in a nonuniform external field, Sov. Phys. JETP 73, 1009.

[28] Shevchenko S. I. (1992) On the theory of a Bose gas in a nonuniform field, Sov. J. Low Temp. Phys. 18, 223.

[29] Kolomeisky E. B., Newman T. J., Straley J. P., and Qi X. (2000) Low-dimensional Bose liquids: beyond the Gross-Pitaevskii approximation, Phys. Rev. Lett. 85, 1146.

[30] Lieb E. H., Seiringer R., and Yngvason J. (2001) A rigorous derivation of the GrossPitaevskii energy functional for a two-dimensional Bose gas, Commun. Math. Phys. 224, 17.

[31] Petrov D. S., Holzmann M., and Shlyapnikov G. V. (2000) Bose-Einstein condensation in quasi-2D trapped gases, Phys. Rev. Lett. 84, 2551.

[32] Petrov D. S. and Shlyapnikov G. V. (2001) Intertomic collisions in a tightly confined Bose gas, Phys. Rev. A 64, 012706.

[33] Lee M. D., Morgan S. A., Davis M. J., and Burnett K. (2002) Energy-dependent scattering and the Gross-Pitaevskii equation in two-dimensional Bose-Einstein condensates, Phys. Rev. A 65, 043617.

[34] Nunes G. S. (1999) Density functional theory of the inhomogeneous Bose-Einstein condensate, J. Phys. B 32, 4293.

[35] Fabrocini A. and Polls A. (1999) Beyond the Gross-Pitaevskii approximation: local density versus correlated basis approach for trapped bosons, Phys. Rev. A 60, 2319.

[36] Kim Y. E. and Zubarev A. L. (2003) Density-functional theory of bosons in a trap, Phys. Rev. A67, 015602.

[37] Tanatar B., Minguzzi A., Vignolo P., and Tosi M. P. (2002) DEnsity profile of a BoseEinstein condensate inside a pancake-shaped trap: observational consequences of the dimensional cross-over in the scattering regime, Phys. Lett. A 302, 131.

[38] Schick M. (1971) Two-dimensional system of hard-core bosons, Phys. Rev. A 3, 1067.

[39] Banerjee A. and Singh M. P. (2001) Ground-state properties of a trapped Bose gas beyond the mean-field approximation, Phys. Rev. A 64, 063604.

[40] Andersen J. O. and Haugerud H. (2001) Ground state of a trapped Bose-Einstein condensate in two dimensions; beyond the mean-field approximation, Phys. Rev. A 65, 033615.

[41] Dalfovo F. and Stringari S. (1996) Bosons in anisotropic traps: ground state and vortices, Phys. Rev. A 53, 2477.

[42] Cornish S. L., Claussen N. R., Roberts J. L., Cornell E. A., and Wiemann C. E. (2000) Stable ${ }^{85} \mathrm{Rb}$ Bose-Einstein condensates with widely tunable interactions, Phys. Rev. Lett. 85, 1795 . 